\title{
High-frequency oscillatory ventilation combined with partial liquid ventilation in experimental lung injury: effects on lung cell apoptosis
}

\author{
Huimin Zuo $\cdot$ Linxiang Zeng · Guanghua Guo · Huihong Zeng
}

Received: 19 April 2014 / Accepted: 19 January 2015 / Published online: 3 April 2015

(C) The Author(s) 2015. This article is published with open access at Springerlink.com

\section{Summary}

Objective To investigate the effects of high-frequency oscillatory ventilation (HFOV) and partial liquid ventilation (PLV) on apoptosis of lung tissue induced by steam inhalation injury in rabbit.

Design A prospective, randomized, controlled, multiple-group study.

Setting An animal research laboratory centre in a university burns centre.

Subjects NewZealand rabbits $(n=32 ; 2.25 \pm 0.25 \mathrm{~kg})$ of either sex.

Interventions The animals were ventilated by HFOV with a mean airway pressure of $10 \mathrm{~cm} \mathrm{H}_{2} \mathrm{O}$, a frequency of $10 \mathrm{~Hz}$, an amplitude of $20 \mathrm{~cm} \mathrm{H}_{2} \mathrm{O}$, an inspiratory:expiratory ratio of 1:1, and an $\mathrm{FiO}_{2}$ of 1.0. After the induction of acute lung injury (ALI) by steam inhalation, the animals were randomly divided into four groups: CMV, HFOV, CMV + PLV, HFOV + PLV group. Then they were ventilated for $4 \mathrm{~h}$ by CMV, HFOV, CMV + PLV and HFOV + PLV, respectively. After the experimental period, cell apoptosis and apoptosis indexes in the lung tissue were assessed with TUNEL FragELTM (Fragment End Labeling).

\section{Dr. L. Zeng $(\bowtie) \cdot$ H. Zuo}

Department of Respiration The Second Affiliated Hospital of Nanchang University,

330006 Nanchang, China

e-mail: zenglinxiang@sohu.com

Dr. L. Zeng

No. 1, Minde Road,

330006 Nanchang, Jiangxi, PR China

\section{G. Guo}

Department of Burns Surgery, The First Affiliated Hospital of Nanchang University, 330006 Nanchang, China

\section{H. Zeng}

Institute for Basic Medical Sciences, Nanchang University, 330006 Nanchang, China
Results Lung tissue apoptosis indexes in HFOV group and HFOV + PLV group were lower than that of in CMV group and CMV + PLV group; between-group comparison had significant difference $(P<0.01)$. HFOV + PLV group showed lowest apoptosis indexes.

Conclusion HFOV combined with PLV can suppress lung tissue apoptosis induced by steam inhalation.

Keywords High-frequency oscillatory ventilation · Partial liquid ventilation - Cell apoptosis · Inhalation injury

\section{Introduction}

Mechanical ventilation is usually necessary to achieve sufficient gas exchange in severe respiratory failure caused by adult respiratory distress syndrome (ARDS) or ALI [1]. Although conventional mode ventilation (CMV) or synchronized intermittent mandatory ventilation with lung protective strategy reduces the complication and improves outcome, however it may still cause mechanical injury to the lungs [2-4]. High-frequency oscillatory ventilation (HFOV), a technique of rapid ventilation with use of very small tidal volume has a potential of reducing ventilator associated lung injuries [5-6], particularly when started early, before significant lung damage has been caused by tidal ventilation [7].

HFOV addresses all of these mechanisms of injury while maintaining adequate gas exchange, and may thereby serve as the optimal mode of ventilation in patients with ARDS [8]. Meta-analysis shows that HFOV can reduce mortality of adult ARDS, reduce intractable hypoxemia, hypotension, barotrauma and incidence of hypercapnia [9]. In paediatric patients with acute respiratory failure, failing conventional ventilation (CV), HFOV can improve hypercapnia and gas exchange in a rapid and sustained fashion [10-11]. It is a safe option for the treatment of ARDS and severe small airway diseases [12]. 
Partial liquid ventilation (PLV) has been shown to markedly improve oxygenation through recruitment of a collapsed alveolar space and reduce histologic evidence of injury in a number of lung injured models [13]. In addition to improving lung function in animal models of ALI, PLV also attenuates the inflammatory response and reduces pulmonary neutrophil accumulation. This phenomenon occurs in numerous models of ALI [14]. HFOV and PLV developed as a rescue and lung-protective ventilation strategy have become major concerns in recent years. HFOV represents an 'open lung' approach to mechanical ventilation that uses tidal volumes less than physiologic dead space and sustained mean airway pressures to maintain lung recruitment. The appropriate mean airway pressure can improve the functional residual capacity, increase the oxygen diffusion area and improve oxygenation state. During HFOV, tidal volume (Vt) and associated swings in alveolar pressure are very small [15-16]. It can improve arterial oxygenation, reduce ventilator-associated lung injury. HFOV achieves oxygenation and ventilation by oscillating the lung around a constant mean airway pressure, with small tidal volumes at a high frequency. Patients with ALI have a high mortality and often require the assistance of mechanical ventilation, which may further aggravate the ALI owing to the risk of barotraumas, polytrauma and bio traumas. Severe steam inhalation injury can cause all mild, moderate and severe type of ARDS; according to the Berlin Definition, is also associated with increased mortality [17].

During the mechanical ventilation period, pulmonary endothelial cells are simultaneously subjected to mechanical stretch and fluid shear because of its function, position and special structure. Mechanical ventilation and pulmonary stretch directly change the configuration of the cell, affect the function of endothelial cells, also make the fluid shearing stress cyclically change with the rhythmic mechanical ventilation, which further influence the endothelial cells [18-21].

The pathogenesis of inhalation injury has not been completely elucidated; studies have shown that cell apoptosis is involved in the pathological process following inhalation injury [22]. For further observations of lung cell apoptosis to inhalation injury, the change of apoptosis after mechanical ventilation therapy and its relationship with apoptosis will help to reveal the essence of inhalation injury.

In our study, we use four modes of ventilation treatment in the rabbit model of steam inhalation injury and acute respiratory failure to observe the changes in ventilation of apoptosis lung tissue and to investigate the treatment mechanism of the high-frequency oscillation ventilation combined PLV on inhalation injury.

\section{Materials and methods}

All the protocols used in this study were in accordance with the guidelines for the care and uses of laboratory animals published by the National Institute of Health in
China and were approved by the Committee of Jiangxi Science Council, Nanchang, China.

\section{Animal preparation and ventilation treatment}

We utilized 32 healthy adult New Zealand white rabbits $(2.25 \pm 0.25 \mathrm{~kg})$ of either sex, adopted the method of the Chinese People's Liberation Army Third Military Medical University [23], to reproduce the rabbits model of steam inhalation injury. When the arterial blood oxygen partial pressure was less than $60 \mathrm{mmHg}$, the model is completed. Then the rabbits were randomly divided into CMV ( $n=8)$, treated with CMV only; CMV + PLV $(n=8)$, treated with a combined therapy of CMV + PLV; HFOV $(n=8)$, treated with CMV only; HFOV + PLV treated with a combined therapy of HFOV + PLV. The CMV and CMV + PLV groups were conducted using CMV with VT $10 \mathrm{ml} /$ $\mathrm{kg}, 30$ breathes/min, inspiratory oxygen fraction $100 \%$, inspiratory time $1 \mathrm{~s}$ and positive end-expiratory pressure (PEEP) $0 \mathrm{~cm} \mathrm{H}_{2} \mathrm{O}$. The HFOV and HFOV + PLV groups were switched to HFOV with mean airway pressure set at $12 \mathrm{~cm} \mathrm{H}_{2} \mathrm{O}$, an $\mathrm{FiO}_{2}$ of 1.0 , a frequency of $10 \mathrm{~Hz}$, an amplitude of $20 \mathrm{~cm} \mathrm{H}_{2} \mathrm{O}$, inspiratory to expiratory ratio of $1: 1$.

Throughout the experiment, heart rate (HR) and mean blood pressure (MBP) was monitored and recorded, and femoral artery blood was withdrawn every 0.5, 1.5, 2.5 and $3.5 \mathrm{~h}$ for arterial blood gas analysis during the mechanical ventilation treatment period.

With its characteristics of high density, perfluorocarbons (PFCs) can be deposited on the prolapse part of lung because of the effect of gravity. A certain volume of liquid can make the atelectatic alveoli to be opened again; its action is similar to "liquid" PEEP. PFC (perfluorodecalin; F2 Chemical's Ltd., Lea Lane, LeDifferent positional Town, Preston, Lancashine, PR4 OPZ, UK) is injected two times via an endotracheal tube at different body positions of mechanical ventilation in CMV + PLV and $\mathrm{HFOV}+\mathrm{PLV}$ groups, initially $1 \mathrm{ml} / \mathrm{kg}$, and $30 \mathrm{~min}$ later, additional $0.5 \mathrm{ml} / \mathrm{kg}$ at a different body position-a total of $3 \mathrm{ml} / \mathrm{kg}$ implemented as PLV.

\section{Apoptosis steps monitoring by TUNEL assay}

Phlebotomize after 4-h ventilation treatment in each experimental group, apoptotic cells in pulmonary tissue from all groups were detected by the FragELTM (Fragment-end-labeling technique). The terminal deoxynucleotidyl transferase-mediated dUTP nick end labeling (TUNEL) assay was performed using an In Situ Apoptosis Detection kit according to the manufacturer's instructions (Promega, Michigan, USA). Briefly, paraffin-embedded sections were deparaffinized with xylene and dehydrated through graded alcohols. After washing with PBS, the slides were permeated with $0.1 \%$ Triton $\mathrm{X}-100$ in $0.1 \%$ sodium citrate for 2 min once. And endogenous peroxidase activity was quenched by incubation of the sections for 10 min with $3 \%$ hydrogen peroxide 
in PBS. Slides were placed in equilibration buffer and then placed in working-strength TdT enzyme, followed by working-strength stop/wash buffer. After two drops of anti-digoxigenin-biotin were applied to the slides, diaminobenzidine was used to detect peroxidase and then washed with deionized water. Counterstaining of nuclei was performed with Immunomaster's Hematoxylin by incubating the sections at room temperature for $10 \mathrm{~min}$. Reactive nuclei that were brownish tinge served as positive controls. Slides without being placed in TdT served as negative controls. The assessments of the number of apoptotic cells by TUNEL assay were performed using captured high-quality images obtained with an image analysis system (HMIAS-2000; Video Technology Co., Ltd, Wuhan, China). The apoptosis index was calculated as: the number of apoptotic cells/total cells $\times 100 \%$, the results are expressed with mean \pm SEM.

\section{Statistical analysis}

The indicators are expressed as the mean \pm standard error of the mean, all data are performed by SPSS12.0 statistics software analysis, Comparisons of each group were made using a paired $t$-test, comparison among the groups were evaluated by analysis of variance. Difference was defined for values of $P<0.05$. Difference was significantly defined for values of $P<0.01$.

\section{Result}

$\mathrm{PaO}_{2}, \mathrm{PaCO}_{2}, \mathrm{HR}$ and MBP significantly change in these animals immediately after four different ventilation treatments.

Changes in arterial blood gases during the $3.5 \mathrm{~h}$ observation period are presented in Table $1 . \mathrm{PaO}_{2}$ displayed a trend of improvement after half an hour ventilation treatment. There were significant changes compared with other periods $(P<0.01)$.

Table 1 showed $\mathrm{PaO}_{2}$ was at a higher level during the whole experiment, after 0.5 - $\mathrm{h}$ of treatment, $\mathrm{PaO}_{2}$ began to rise, all groups had their best $\mathrm{PaO}_{2}$ at the $2.5 \mathrm{~h}$ time point. HFOV showed better results with $\mathrm{PaO}_{2}$ over time versus the CMV group; CMV + PLV group also had the $\mathrm{PaO}_{2}$ increase after 0.5-h treatment. Both the HFOV and the CMV + PLV groups showed better results with $\mathrm{PaO}_{2}$ over time versus the CMV group. HFOV + PLV showed significantly better results with $\mathrm{PaO}_{2}$ compared with the other three groups, after 3.5-h treatment, HFOV + PLV HFOV had better results of $\mathrm{PaO}_{2}$. The values of $\mathrm{PaO}_{2}$ with HFOV + PLV group were significantly higher than the $\mathrm{CMV}+\mathrm{PLV}$ group after 2.5 and $3.5 \mathrm{~h}$ treatment.

Table 2 showed the changes in HR (MBP) during the 3.5-h observation period. After four groups of different ventilation mode treatment, the HR remained fairly stable over time, changes within the group and between groups have no statistical significance $(P>0.05)$.
Table 1 Changes in arterial $\mathrm{PaO}_{2}$ over time in the various treatment groups ( $\mathrm{mmHg} \bar{X} \pm \mathrm{s}, n=8$ )

\begin{tabular}{|c|c|c|c|c|c|}
\hline Group & $0 \mathrm{~h}$ & $0.5 \mathrm{~h}$ & $1.5 \mathrm{~h}$ & $2.5 \mathrm{~h}$ & $3.5 \mathrm{~h}$ \\
\hline $\mathrm{CMV}^{\mathrm{a}}$ & $58 \pm 4$ & $209 \pm 50$ & $175 \pm 47$ & $187 \pm 53$ & $179 \pm 55$ \\
\hline $\mathrm{CMV}+\mathrm{PLV}^{\mathrm{b}}$ & $55 \pm 9$ & $213 \pm 44$ & $238 \pm 42$ & $243 \pm 49$ & $186 \pm 50$ \\
\hline $\mathrm{HFOV}^{\mathrm{c}}$ & $576 \pm 7$ & $209 \pm 38$ & $239 \pm 36$ & $272 \pm 41$ & $215 \pm 38$ \\
\hline HFOV + PLV ${ }^{d}$ & $55 \pm 7$ & $214 \pm 51$ & $269 \pm 45$ & $291 \pm 39$ & $277 \pm 42$ \\
\hline
\end{tabular}

CMV conventional mode ventilation, HFOV high-frequency oscillatory ventilation alone, HFOV + PLV combined therapy of HFOV + PLV, PLV partial liquid ventilation

$0.5 \mathrm{~h}$ time point between group analysis: $P>0.05$

$1.5 \mathrm{~h}$ time point between group $a$ and $b, a$ and $c$ analysis: $P<0.05, a$ and $d$ analysis: $P<0.01$; between other groups analysis: $P>0.05$

$2.5 \mathrm{~h}$ time point between group $a$ and $b$ analysis: $P<0.05, a$ and $c, a$ and $d$ analysis: $P<0.01, b$ and $d$ analysis: $P<0.01, c$ and $b, c$ and $d$ analysis: $P>0.05$

$3.5 \mathrm{~h}$ time point between $d$ and other groups analysis: $P<0.01$ between other groups analysis: $P>0.05$

Data are expressed as the mean \pm SEM

Table 2 Changes in heart rate $(\mathrm{HR})$ in various treatment groups ( $\bar{X} \pm \mathrm{s}, n=8)$

\begin{tabular}{|c|c|c|c|c|c|}
\hline Group & $0 \mathrm{~h}$ & $0.5 \mathrm{~h}$ & $1.5 \mathrm{~h}$ & $2.5 \mathrm{~h}$ & $3.5 \mathrm{~h}$ \\
\hline $\mathrm{CMV}^{\mathrm{a}}$ & $286 \pm 18$ & $286 \pm 13$ & $281 \pm 14$ & $280 \pm 14$ & $284 \pm 16$ \\
\hline $\mathrm{CMV}+\mathrm{PLV}^{\mathrm{b}}$ & $281 \pm 13$ & $278 . \pm 16$ & $282 \pm 13$ & $281 \pm 17$ & $285 \pm 16$ \\
\hline $\mathrm{HFOV}^{\mathrm{c}}$ & $286 \pm 20$ & $284 \pm 17$ & $283 \pm 15$ & $286 \pm 18$ & $283 \pm 15$ \\
\hline $\mathrm{HFOV}+\mathrm{PLV}^{\mathrm{d}}$ & $286 \pm 18$ & $287 \pm 21$ & $283 \pm 16$ & $282 \pm 17$ & $286 \pm 14$ \\
\hline \multicolumn{6}{|c|}{$\begin{array}{l}C M V \text { conventional mode ventilation, } H F O V \text { high-frequency oscillatory } \\
\text { ventilation alone, } H F O V+P L V \text { combined therapy of HFOV }+P L V, P L V \text { partial } \\
\text { liquid ventilation } \\
\text { Within-group analysis: } P>0.05 \text { vs post-injury. Between-group analysis: } \\
P>0.05 \\
\text { Data are expressed as the mean } \pm \text { SEM }\end{array}$} \\
\hline
\end{tabular}

Table 3 Apoptosis index in lung tissue $(\bar{X} \pm \mathrm{s}, n=8)$

\begin{tabular}{l|l}
\hline Group & Apoptosis indexes (\%) \\
\hline CMV & $39.57 \pm 6.36$ \\
\hline CMV + PLV & $25.83 \pm 7.15$ \\
\hline HFOV & $21.64 \pm 3.80$ \\
\hline HFOV + PLV & $13.52 \pm 5.33$ \\
\hline $\begin{array}{l}\text { CMV conventional mode ventilation, HFOV high-frequency oscillatory } \\
\text { ventilation alone, HFOV + PLV combined therapy of HFOV + PLV, PLV partial }\end{array}$ \\
$\begin{array}{l}\text { liquid ventilation } \\
\text { Apoptosis indexes: Between HFOV and CMV + PLV, HFOV and HFOV + PLV } \\
\text { analysis }(P>0.05), \text { Between other groups analysis }(P<0.01)\end{array}$
\end{tabular}

However, the values of $\mathrm{PaCO}_{2}$ in four groups have increased after induction of ALI, but fluctuated with $30 \sim 42 \mathrm{mmHg}$ during the ventilatory treatment, there are no significant changes within the group and between groups. The changes of MBP during the 3.5-h observation period within the group and between groups have no statistical significance $(P>0.05)$ after four groups of different ventilation mode treatment.

Table 3 showed lung tissue apoptosis index of HFOV + PLV group is lowest, as $13.52 \%$, CMV group is highest, as $39.57 \%$. HFOV and HFOV + PLV group were significantly 
Fig. 1 Lung tissue cell apoptosis $(\times 200)$ of rabbits with steam inhalation injury in the various modes of ventilatory therapy were assessed with TUNEL FragELTM. CMV conventional mode ventilation, HFOV high-frequency oscillatory ventilation alone, $P L V$ partial liquid ventilation

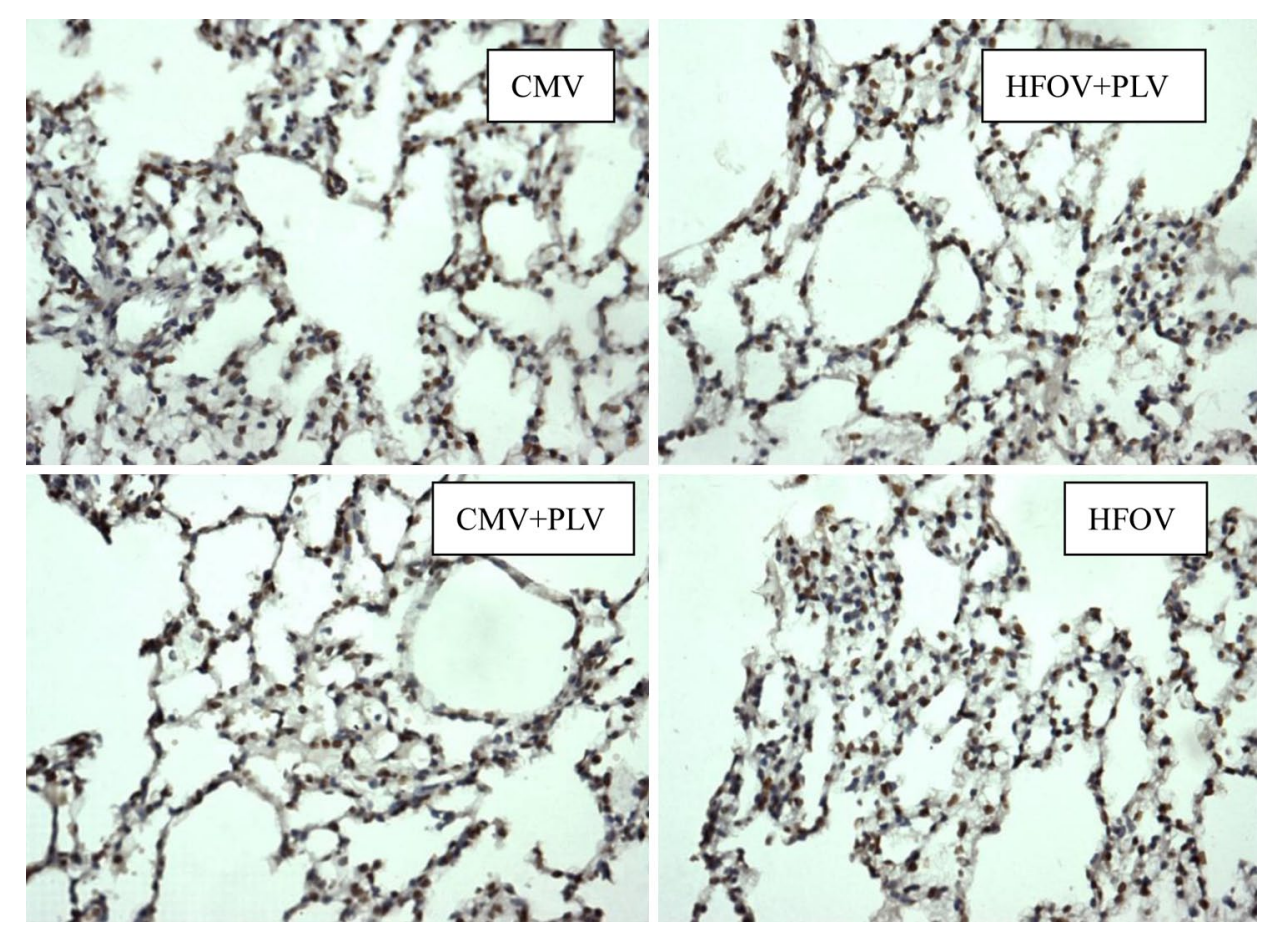

lowered than of the CMV and CMV + PLV group, respectively comparison between groups had significant difference $(P<0.01)$. And CMV + PLV group is lower than CMV group without PLV, compare between groups had significant difference $(P<0.01)$; HFOV + PLV group is lower than HFOV group, but there was no significant difference $(P>0.05)$.

TUNEL-positive staining nuclei emerge tan; karyopyknosis emerge rounded (Fig. 1). Some irregular chromatin is gathered together and distributed on the brink of nucleus, which conforms to the morphology of apoptotic cells; apoptotic body can be found in several cells.

\section{Discussion and conclusions}

Apoptosis is a programmed cell death; cell apoptosis is closely associated with inhalation injury [24]. Apoptosis participates in inhalation injury, and the pathological process of ventilator-associated lung injury [25], inhibiting the excessive apoptosis of lung tissue cells can reduce lung injury; it may be a new way of lung injury prevention. Steam inhalation injury can accelerate lung tissue apoptosis; its mechanism is related to the following factors: (1) the tissue is ischaemic and hypoxic; (2) inflammatory mediators, cytokines, oxygen, free radicals, reactive oxygen species, metabolic intermediate, etc., directly or indirectly, interfere with apoptosis control gene expression, and promote cell apoptosis; (3) thermodynamic effects of the steam. Mechanical ventilation can improve alveolar ventilation function, which is an important means of treatment of severe burns complicated by inhalation injury, but mechanical ventilation can cause excessive tension in atelectatic lung alveolar tissue; after repeatedly being closed and opened, the mechanical ventilation and shearing damage induced the release of inflammatory mediators, and also can accelerate vascular endothelial cells and alveolar epithelial cell apoptosis [26]. Hammerschmidt experiment confirmed that excessive tension can directly cause alveolar epithelial cell apoptosis [27]. While the conclusion is the opposite in Mr. Imai and Fisher reports: Despite a high degree of invasive mechanical ventilation, the number of apoptosis is reduced [28]. The exact mechanism of effect of mechanical ventilation on the apoptosis will be further investigated in the future.

To understand different mechanical ventilation treatment with rabbit induced by steam inhalation injury, the mechanism and function pathway of effect on apoptosis of lung tissue, we adopt TUNEL technique to monitor lung tissue apoptosis. The ventilation experiment HFOV + PLV treatment group apoptosis index is the lowest, CMV group apoptosis index is the highest. Apoptosis index of HFOV group and HFOV + PLV group is significantly less than that of CMV and CMV + PLV group, which shows that high-frequency oscillation ventilation can reduce lung tissue apoptosis in rabbit induced by steam inhalation injury, which is due to the characteristics of HFOV, high-frequency, low tidal volume, and a constant, less variable airway pressure can alleviate mechanical stimulation of the tension stress and shearing stress of lung tissue, avoid the alveolar epithelial cells stretching by excessive force, therefore, it reduces the occurrence of apoptosis. In this experiment, cell apoptosis of CMV + PLV group and HFOV + PLV is less than CMV group and HFOV group, showing that PLV can decrease the rate of lung tissue apoptosis, the mechanism may be due to the following ways: (1) the PFC can improve lung of 
respiratory function, correct the cell hypoxia by reducing alveolar surface tension and dead space, improving V/Q matching; (2) the fluorine carbon can reduce the concentration of adhesion molecules and mRNA expression in the lungs, thus reducing the neutrophils' adhesion to vascular endothelial cell and detention in the lungs, suppress the inflammatory reaction of lung tissue, thus reduce the inflammation mediums; stability of cell membrane and suppress lung inflammatory mediators and cytokines release; (3) the fluorine carbon may regulate gene expression by influencing apoptosis, reduce the apoptosis of lung tissue cells.

However, our results lacked tissue cell types and had a major problem with ventilator settings using high tidal volumes without PEEP as controls (CMV and CMV + PLV) for HFOV. There are two reasons: First, depending on the characteristics of high density, PFCs can be deposited on the prolapse of part of lung because of the effect of gravity. A certain volume of liquid can make the atelectatic alveolar to be opened again; its function is similar to "liquid" PEEP. To exclude the intervention of PEEP and indicate the effect of "liquid" PEEP, we performed without PEEP. Second, meta-analysis evidence indicates that high levels of PEEP, as compared with low levels, did not reduce mortality before hospital discharge. The data also show that high levels of PEEP produced no significant difference in the risk of barotrauma [29]. But this is not in accordance with actual clinical recommendations of a lung protective ventilation strategy for ARDS patients. Patients were encouraged to use pressure-controlled ventilation at 6-8 $\mathrm{ml}$ per kilogram of ideal body weight and to use the combinations of PEEP and $\mathrm{FiO}_{2}$ values that were used in the Acute Respiratory Distress Syndrome Network study in the CV strategy [30]. We recommend that this mode of ventilation not to be used for routine care.

Regardless of its limitations, this study is interesting and suggests that lung tissue is injured after inhalation steam and that HFOV with PLV strategy reduces lung cells' damage than CV although this setting is not in accordance with international recommendations for ventilation of ARDS patients. Further, more research analysis is needed and must be broadened to study the possible mechanisms of HFOV to minimize injury.

We obtained positive results using a combination therapy of HFOV and PLV to treat experimental lung injury induced by steam inhalation compared with conventional mechanical ventilation. This experiment demonstrates HFOV and PLV reduce the lung tissue apoptosis, and have the synergy with each other.

During 2013, two large randomized controlled trials comparing HFOV with a conventional lung-protective ventilation were published-the Oscillation for Acute Respiratory Distress Syndrome Treated Early (OSCILLATE) and the Oscillation in ARDS (OSCAR) trials. These trials suggested no benefit or even harm with HFOV in adults with early moderate-to-severe ARDS [31,32]. There might be a treatment benefit of HFOV over CV in patients with ARDS and higher baseline the oxygenation index [33]. A study shows liquid ventilation using PFCs provide gas exchange in the lungs, potentially useful adjunct in the management of severe respiratory failure [34].

Many animal studies indicated that PLV can alleviate the histological damage of ALI induced by meconium aspiration (MAS) and increased survival chance and therefore PLV would be a useful treatment for MAS [35]. And PLV with PFCs may protect the lung from acute pulmonary inflammation more effectively than CV or HFOV does [36].

Future studies on combined therapy with HFOV + PLV will need to identify those patients who might benefit most from HFOV and to determine the best oscillator settings. Both goals require an improved capability of monitoring recruitment and over distension, and oscillatory volumes. Moreover, future directions with potential new technical advances and the use of new bedside monitoring techniques needed to be addressed.

\section{Conflict of interest}

The authors report that this study did not receive any financial and personal relationships with other people or organizations that could inappropriately influence our work. There are no potential conflicts of interest.

\section{Open Access}

This article is distributed under the terms of the Creative Commons Attribution License which permits any use, distribution, and reproduction in any medium, provided the original author(s) and the source are credited.

\section{References}

1. International Consensus Conferences in Intensive Care Medicine. Ventilator-associated lung injury in ARDS. Am J Respir Crit Care Med. 1999;160:2118-24.

2. Oxford Region Controlled Trial of Artificial Ventilation OCTAVE Study Group. Multicentre randomised controlled trial of high against low frequency positive pressure ventilation. Arch Dis Child. 1991;66:770-5.

3. The Acute Respiratory Distress Syndrome Network. Ventilation with lower tidal volumes as compared with traditional tidal volumes for acute lung injury and the acute respiratory distress syndrome. N Engl J Med. 2000;342:1301-8.

4. Dreyfuss D, Saumon G. Ventilator-induced lung injury: lessons from experimental studies. Am J Respir Crit Care Med. 1998;157:294-323

5. Yoder RA, Siler-Khodr T, Winter VT, Coalson JJ. High frequency oscillatory ventilation: effects on lung function, mechanics, and airway cytokines in the immature baboon model for neonatal chronic lung disease. Am J Respr Crit Care Med. 2000;162:1867-76.

6. Hamilton PP, Onayerni A, Smith JA, Gillan JE, Cutz E, Froese $A B$, et al. Comparison of conventional and high frequency ventilation: oxygenation and lung physiology. J Appl Physiol. 1983;55:131-8.

7. deLemos RA, Coolson JJ, Meredith KS, Gerstmann DR, Null DM. A comparison of ventilation strategies for the use of high-frequency oscillatory ventilation in the treatment of hyaline membrane disease. Acta Anaesthesiol Scand. 1989;33:102-7. 
8. Ferguson ND, Slutsky AS. Point: counterpoint: high frequency ventilation is/is not the optimal physiological approach to ventilate ARDS patients. J Appl Physiol. 2008;104(4):1230-1.

9. Sud S, Sud M, Friedrich JO, Meade MO, Ferguson ND, Wunsch $\mathrm{H}$, et al. High frequency oscillation in patients with acute lung injury and acute respiratory distress syndrome (ARDS): systematic review and meta-analysis. BMJ. 2010;340(1):c2327-38.

10. Ben Jaballah N, Khaldi A, Mnif K, Bouziri A, Belhadj S, Hamdi A, Kchaou W. High-frequency oscillatory ventilation in pediatric patients with acute respiratory failure. Pediatr Crit Care Med. 2006;7(4):362-7.

11. Pinzon AD, Rocha TS, Ricachinevsky C, Piva JP, Friedman G. High-frequency oscillatory ventilation in children with acute respiratory distress syndrome: experience of a pediatric intensive care unit. Rev Assoc Med Bras. 2013;59(4):368-74.

12. Moniz M, Silvestre C, Nunes P, Abadesso C, Matias E, Loureiro H, Almeida H. High-frequency oscillatory ventilation in children: a 10-year experience. J Pediatr (Rio J). 2013;89(1):48-55.

13. Kaisers U, Kelly KP, Busch T. Liquid ventilation. Br J Anaesth. 2003;91:143-51.

14. Colton DM, Till GO, Johnson KJ, Dean SB, Bartlett RH, Hirschl RB. Neutrophil accumulation is reduced during partial liquid ventilation. Crit Care Med. 1998;26:1716-24.

15. Bohn DJ, Miyasaka K, Marchak BE, Thompson WK, Froese $\mathrm{AB}$, Bryan AC. Ventilation by high-frequency oscillation. J Appl Physiol. 1980;48:710-6.

16. Slutsky AS. Mechanisms affecting gas transport during high frequency oscillations. Crit Care Med. 1984;12:713-7.

17. ARDS Definition Task Force, Ranieri VM, Rubenfeld GD, et al. Acute respiratory distress syndrome: the Berlin Definition. JAMA. 2012;307(23):2526-33.

18. Verbrugge SJC, Lachmann B, Kesecioglu J. Lung protective ventilatory strategies in acute lung injury and acute respiratory distress syndrome: from experimental findings to clinical application. Clin Physiol Funct Imaging. 2007;27:67-90.

19. David M, Heinrichs W. High frequency oscillatory ventilation and an interventional lung assist device to treat hypoxaemia and hypercapnia. Br J Anaesth. 2004;93(4):582-6.

20. Dembinski R, Max M, Bensberg R, et al. High frequency oscillatory ventilation in experimental lung injury: effects on gas exchange. Intensive Care Med. 2002;28(6):768-74

21. Meinhardta JP, Friessa U, Bendera HJ. Relationship among cardiac index, inspiration/expiration ratio, and perfluorocarbon dose during partial liquid ventilation in an oleic acid model of acute lung injury in sheep. J Pediatr Surg. 2005;40:1395-403.

22. Wenjun L, Zongcheng Y, Xiaodong Y, et al. Apoptosis and changes in apoptosis regulating genes in lung tissue cells after smoke inhalation injury in rats. Med J Chinese People's Liberation Army. 2002;27(2):142-4.

23. Zhu Peifang First Affiliated Hospital. A canine model of severe steam respiratory burns. Med J Chinese People's Liberation Army. 1983;8(1):7-10.
24. Bem RA, Bos AP, Matute Bello G, et al. Lung epithelial cell apoptosis during acute lung injury in infancy. Pediatr Crit Care Med. 2007;8(2):132-7.

25. Saito S, Ogama J, Minamiya Y. Pulmonary reexpansion causes xanthine oxidase-induced apoptosis in rat lung. Am J Physiol Lung Cell Mol Physiol. 2005;289(3):400-6.

26. Hammerschmidt S, Kuhn H, Gessner C, et al. Stretchinduced alveolar type II cell apoptosis: role of endogenous bradykinin and PI3K-Akt signaling. Am J Respir Cell Mol Biol. 2007;37:699-705.

27. Hammerschmidt S, Kuhn H, Grasenack T, et al. Apoptosis and necrosis induced by cyclic mechanical stretching in alveolar type II cells. Am J Respir Cell Mol Biol. 2004;30:396-402.

28. Imai Y, Parodo J, Kajikawa O, et al. Injurious mechanical ventilation and end-organ epithelial cell apoptosis and organ dysfunction in an experimental model of acute respiratory distress syndrome. JAMA. 2003;289:2104-12.

29. Santa Cruz R, Rojas JI, Nervi R, Heredia R, Ciapponi A. High versus low positive end-expiratory pressure (PEEP) levels for mechanically ventilated adult patients with acute lung injury and acute respiratory distress syndrome. Cochrane Database Syst Rev. 2013;(6):CD009098. doi:10.1002/14651858.CD009098.pub2.

30. The Acute Respiratory Distress Syndrome Network. Ventilation with lower tidal volumes as compared with traditional tidal volumes for acute lung injury and the acute respiratory distress syndrome. N Engl J Med. 2000;342:1301-8.

31. Goffi A, Ferguson ND. High-frequency oscillatory ventilation for early acute respiratory distress syndrome in adults. Curr Opin Crit Care. 2014;20(1):77-85.

32. Young D, Lamb SE, Shah S. High-frequency oscillation for acute respiratory distress syndrome. $\mathrm{N}$ Engl J Med. 2013;368:806-13.

33. Bollen CW, van Well GTJ, Sherry T, et al. High frequency oscillatory ventilation compared with conventional mechanical ventilation in adult respiratory distress syndrome: a randomized controlled trial [ISRCTN24242669]. Crit Care. 2005;9:R430-9.

34. Brown JK, Haft JW, Bartlett RH, Hirschl RB. Acute lung injury and acute respiratory distress syndrome: extracorporeal life support and liquid ventilation for severe acute respiratory distress syndrome in adults. Semin Respir Crit Care Med. 2006;27(4):416-25.

35. Zhu XD, Chen F, Ji RX, DI H, Xie LJ, Zhu JX. Pathological change of partial liquid ventilation in a rabbit meconium aspiration lung injury model. Zhonghua Er Ke Za Zhi. 2008;46(10):774-8.

36. Merz U, Klosterhalfen B, Häusler M, Kellinghaus M, Peschgens T, Hörnchen H. Partial liquid ventilation reduces release of leukotriene B4 and interleukin-6 in bronchoalveolar lavage in surfactant-depleted newborn pigs. Pediatr Res. 2002;51(2):183-9. 